Pacific

Journal of

Mathematics

AN EXPLICIT EXAMPLE OF RIEMANN SURFACES WITH LARGE BOUNDS ON CORONA SOLUTIONS

BYUNG-GEUN OH

Volume $228 \quad$ No. 2

December 2006 


\title{
AN EXPLICIT EXAMPLE OF RIEMANN SURFACES WITH LARGE BOUNDS ON CORONA SOLUTIONS
}

\author{
BYUNG-GEUN OH
}

\begin{abstract}
By modifying Cole's example, we construct explicit Riemann surfaces with large bounds on corona solutions in an elementary way.
\end{abstract}

\section{Introduction}

For a given Riemann surface $R$, consider the algebra $H^{\infty}(R)$ of bounded analytic functions on $R$ separating the points in $R$. The corona problem asks whether $\iota(R)$ is dense in the maximal ideal space $M(R)$ of $H^{\infty}(R)$, where $\iota: R \rightarrow M(R)$ is the natural inclusion defined by the point evaluation. If $\iota(R)$ is dense in $M(R)$, we say that the corona theorem holds for $R$. Otherwise $R$ is said to have corona.

The corona theorem holds for $R$ if and only if the following statement is true (see [Gamelin 1978, Chapter 4] or [Garnett 1981, Chapter VIII]): for every collection $F_{1}, \ldots, F_{n} \in H^{\infty}(R)$ and any $\delta \in(0,1)$ with the property

$$
\delta \leq \max _{j}\left|F_{j}(\zeta)\right| \leq 1 \quad \text { for all } \zeta \in R,
$$

there exist $G_{1}, \ldots, G_{n} \in H^{\infty}(R)$ such that

$$
F_{1} G_{1}+F_{2} G_{2}+\cdots+F_{n} G_{n}=1 .
$$

We refer to $G_{1}, \ldots, G_{n}$ as corona solutions, $F_{1}, \ldots, F_{n}$ as corona data, and $\max \left\{\left\|G_{1}\right\|, \ldots,\left\|G_{n}\right\|\right\}$ as a bound on the corona solutions or corona constant. Here the notation $\|\cdot\|$ indicates the uniform norm. Throughout this paper, we assume that the corona data satisfies (1-1) for the given $\delta$. The letter $\delta$ is reserved only for this use.

Theorem 1 (B. Cole; see [Gamelin 1978, Theorem 4.1, pp. 47-49]). For any $\delta \in(0,1)$ and $M>0$, there exist a finite bordered Riemann surface $R$ and corona data $F_{1}, F_{2} \in H^{\infty}(R)$ such that any corona solutions $G_{1}, G_{2} \in H^{\infty}(R)$ have a bound at least $M$; that is, $\max \left\{\left\|G_{1}\right\|,\left\|G_{2}\right\|\right\} \geq M$.

MSC2000: 30H05, 30D55.

Keywords: corona problem, bounded analytic function. 
The purpose of this paper is to construct the Riemann surface $R$ in Theorem 1 in an elementary way and describe it explicitly. Once Theorem 1 is proved, it is possible to construct a Riemann surface with corona.

Theorem 2 (B. Cole; see [Gamelin 1978, Theorem 4.2, pp. 49-52]). There exists an open Riemann surface with corona.

The basic idea of the proof of Theorem 2 is that if a Riemann surface $R$ is obtained by connecting two Riemann surfaces $R_{1}$ and $R_{2}$ with a thin strip, then any holomorphic function on $R$ behaves almost independently on $R_{1}$ and $R_{2}$.

The corona theorem holds for the unit disc [Carleson 1962], finitely connected domains in $\mathbb{C}$ [Gamelin 1970], Denjoy domains [Garnett and Jones 1985], and various other classes of planar domains and Riemann surfaces [Alling 1964; Behrens 1970; 1971; Jones and Marshall 1985; Stout 1965]. On the other hand, examples of Riemann surfaces with corona (other than Cole's) can be found in [Barrett and Diller 1998] and [Hayashi 1999]. Furthermore, by modifying the proof of Theorem 2, Cole's example can be used to obtain a Riemann surface $R$ with corona that is of Parreau-Widom type [Nakai 1982]. (This means that $\sum_{z \in E} G(z, w)<+\infty$, where $G(\cdot, w)$ is the Green's function on $R$ with the pole $w$ and $E=\{z: \nabla G(z, w)=0\}$.)

The corona problem for a general domain in $\mathbb{C}$ is still open, and the answer is also unknown for a polydisc or a unit ball in $\mathbb{C}^{n}$, for $n \geq 2$.

\section{Proof of Theorem 1}

For given $\delta \in(0,1)$ and $M>0$, we choose a natural number $n$ such that $\delta^{n} \leq$ $\min \left\{(16 M)^{-1}, \frac{1}{4}\right\}$. Let $d=4 \delta^{n^{2}+n}$ and $c=2 \delta^{n^{2}}$. Since $2 \delta^{n^{2}}<2 \delta^{n} \leq \frac{1}{2}$, we have

$$
\frac{4 \delta^{n+1}}{1-c} \leq 8 \delta^{n+1}<8 \delta^{n} \leq \frac{1}{2 M} .
$$

Moreover,

$$
\frac{d}{c-d}=\frac{4 \delta^{n^{2}+n}}{2 \delta^{n^{2}}-4 \delta^{n^{2}+n}}=\frac{2 \delta^{n}}{1-2 \delta^{n}} \leq 4 \delta^{n}<\frac{1}{2 M} .
$$

The important features in our choice of $c, d$ and $n$ are that $d^{1 / n}$ is small (equation $(2-1)), d / c$ is small (equation (2-2)), and $(d / c)^{1 / n}$ is not small - say greater than $\delta$.

Let $\mathbb{D}$ be the unit disc in $\mathbb{C}, B:=B(0, d)=\{z \in \mathbb{C}:|z|<d\}$, and $A:=\mathbb{D} \backslash \bar{B}$. Further, define

$$
\begin{aligned}
& D:=\{z:(z+c) /(1+c z) \in A\}, \\
& D_{1}:=\left\{z: z^{n} \in A\right\}=\left\{z: d / z^{n} \in A\right\}, \\
& D_{2}:=\left\{z: z^{n^{2}} \in D\right\} .
\end{aligned}
$$


Thus $D$ is the image of $A$ under the Möbius transformation $L(z):=(z-c) /(1-c z)$, and $D_{1}$ and $D_{2}$ are preimages of $A$ and $D$ under $h_{1}(z):=d / z^{n}$ and $h_{2}(z):=z^{n^{2}}$. Finally we define the bordered Riemann surface

$$
R:=\left\{\left(z_{1}, z_{2}\right) \in \mathbb{C}^{2}: z_{1} \in D_{1}, z_{2} \in D_{2} \text { and } \frac{z_{1}^{n}-c}{1-c z_{1}^{n}}=z_{2}^{n^{2}}\right\} .
$$

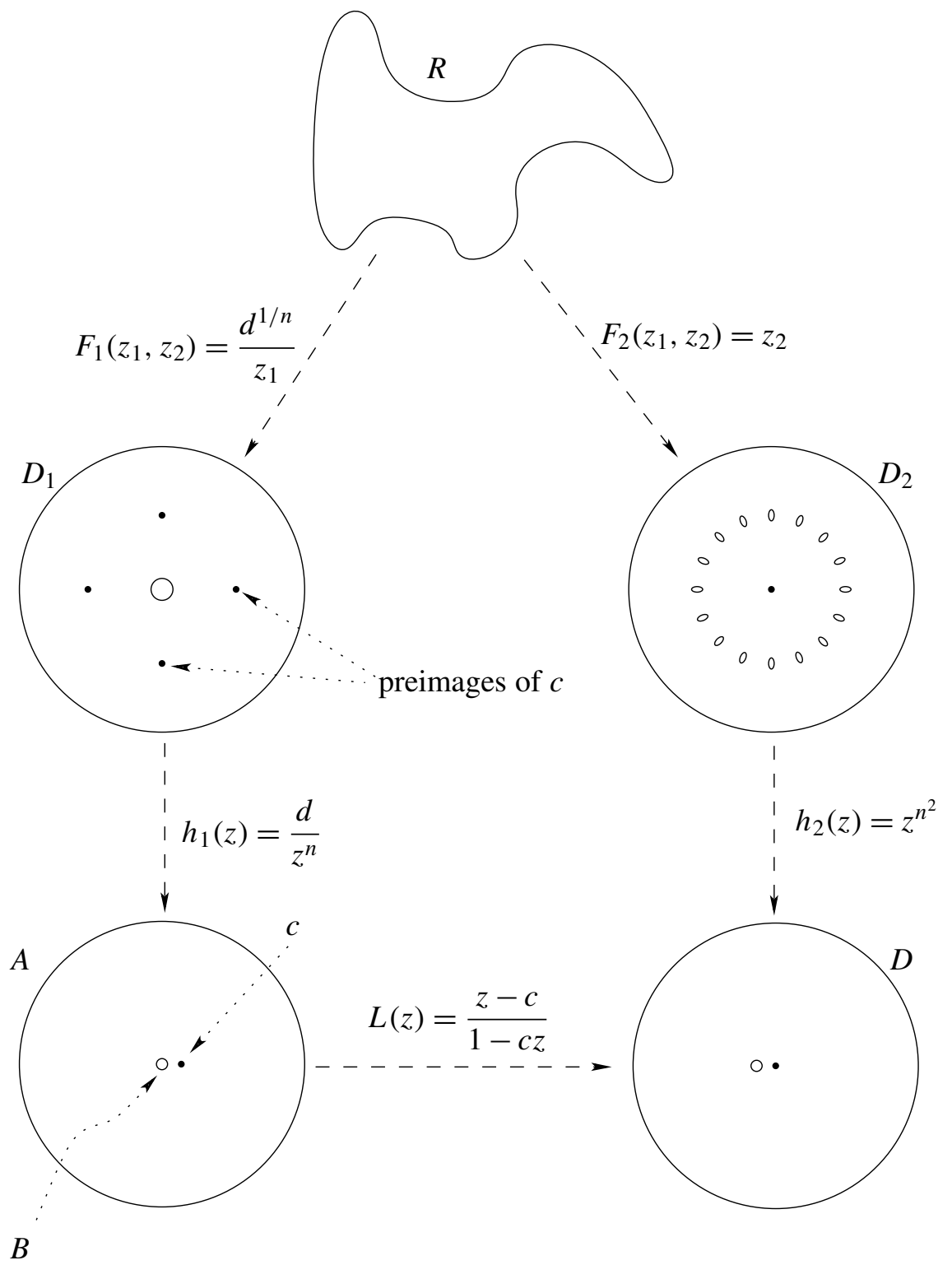

Scheme of the construction of $R$, for $n=4$. 
Note that $R$ is an $n$-sheeted covering of $D_{2}$ and an $n^{2}$-sheeted branched covering of $D_{1}$. This is because $D_{1}$ is an $n$-sheeted covering of $A$ and $D_{2}$ is an $n^{2}$-sheeted branched covering of $D$.

We claim that the Riemann surface $R$, together with the holomorphic functions

$$
F_{1}\left(z_{1}, z_{2}\right)=\frac{d^{1 / n}}{z_{1}} \quad \text { and } \quad F_{2}\left(z_{1}, z_{2}\right)=z_{2}
$$

satisfies the conditions in Theorem 1.

First, note that $F_{1}$ and $F_{2}$ have values in $D_{1}$ and $D_{2}$, respectively. Thus we have $\max \left\{\left\|F_{1}\right\|,\left\|F_{2}\right\|\right\} \leq 1$. Furthermore, if $\left|F_{2}\left(z_{1}, z_{2}\right)\right|=\left|z_{2}\right|<\delta$, we have

$$
\left|z_{1}^{n}-c\right|=\left|z_{2}\right|^{n^{2}}\left|1-c z_{1}^{n}\right|<2 \delta^{n^{2}}
$$

and hence $\left|z_{1}\right|^{n}<c+2 \delta^{n^{2}}=4 \delta^{n^{2}}$. Therefore

$$
\left|F_{1}\left(z_{1}, z_{2}\right)\right|=\frac{d^{1 / n}}{\left|z_{1}\right|}>\frac{4^{1 / n} \delta^{n+1}}{4^{1 / n} \delta^{n}}=\delta
$$

and the inequality $\max \left\{\left|F_{1}\left(z_{1}, z_{2}\right)\right|,\left|F_{2}\left(z_{1}, z_{2}\right)\right|\right\} \geq \delta$ holds for all $\left(z_{1}, z_{2}\right) \in R$; i.e., $\left(F_{1}, F_{2}\right)$ becomes a pair of corona data for the given $\delta$.

It remains to show that $\max \left\{\left\|G_{1}\right\|,\left\|G_{2}\right\|\right\} \geq M$ for any corona solutions $G_{1}, G_{2}$ such that

$$
F_{1} G_{1}+F_{2} G_{2}=1
$$

In fact, we will show that $\left\|G_{1}\right\| \geq M$. To prove this claim, we assume, without loss of generality, that $G_{1}$ and $G_{2}$ are holomorphic across the boundary of $R$. Then we define for all $z \in A$,

$$
f(z):=\frac{1}{n^{3}} \sum F_{1}\left(z_{1}, z_{2}\right) G_{1}\left(z_{1}, z_{2}\right)
$$

where the summation is over all the points $\left(z_{1}, z_{2}\right) \in R$ such that $z_{1}^{n}=z$, counting multiplicity. (Note that the map $\left(z_{1}, z_{2}\right) \mapsto z_{1}^{n}$ is an $n^{3}$-sheeted branched covering from $R$ to $A$.) Then $f$ is analytic in (a neighborhood of) $A$.

Since $F_{2}\left(z_{1}, z_{2}\right)=z_{2}=0$ when $z_{1}^{n}=c$, it is easy to see from (2-4) that $f(c)=1$. On the other hand, $|f(z)| \leq\left\|G_{1}\right\|$ for all $z \in A$ since $\left\|F_{1}\right\| \leq 1$, and $|f(z)| \leq$ $4 \delta^{n+1}\left\|G_{1}\right\|$ for $|z|=1$ since on $\left\{\left|z_{1}\right|=1\right\}$ we have

$$
\left|F_{1}\left(z_{1}, z_{2}\right)\right|=\frac{d^{1 / n}}{\left|z_{1}\right|}=4^{1 / n} \delta^{n+1} \leq 4 \delta^{n+1} .
$$


Therefore, by Cauchy's integral formula,

$$
\begin{aligned}
1=|f(c)| & =\left|\frac{1}{2 \pi i} \int_{|\xi|=1} \frac{f(\xi)}{\xi-c} d \xi-\frac{1}{2 \pi i} \int_{|\xi|=d} \frac{f(\xi)}{\xi-c} d \xi\right| \\
& \leq \frac{4 \delta^{n+1}\left\|G_{1}\right\|}{1-c}+\frac{2 \pi d\left\|G_{1}\right\|}{2 \pi(c-d)} .
\end{aligned}
$$

This inequality, together with (2-1) and (2-2), proves the claim. This completes the proof.

\section{Further remarks}

1. In the construction of $R$, one can take $F_{1}$ as the projection map $\left(z_{1}, z_{2}\right) \mapsto z_{1}$, but then it is necessary to modify the definition (2-3) of $R$ to

$$
R:=\left\{\left(z_{1}, z_{2}\right) \in \mathbb{C}^{2}: z_{1} \in D_{1}, z_{2} \in D_{2} \text { and } \frac{d / z_{1}^{n}-c}{1-c d / z_{1}^{n}}=z_{2}^{n^{2}}\right\}
$$

because we want to make the pair $\left(F_{1}, F_{2}\right)$ a set of corona data satisfying (1-1).

2. Consider the function $h(z):=z^{n}$ defined on $D_{1}$. It is not difficult to see that the Riemann surface $R$ constructed in Section 2 is nothing but the Riemann surface of the multivalued function

$$
h^{-1} \circ L^{-1} \circ h_{2}(z)=\left(\frac{z^{n^{2}}+c}{1+c z^{n^{2}}}\right)^{1 / n}
$$

defined on $D_{2}$. This function takes values in $D_{1}$. Similarly, one can consider $R$ as a Riemann surface of the multivalued function

$$
h_{2}^{-1} \circ L \circ h(z)=\left(\frac{z^{n}-c}{1-c z^{n}}\right)^{1 / n^{2}}
$$

defined on $D_{1}$.

3. We can construct $R$ by cutting and pasting. For example, we can construct the Riemann surface of $h^{-1} \circ L^{-1} \circ h_{2}$ over $D_{2}$ in the following way: we make $n^{2}$ cuts on $D_{2}$ radially so that each cut connects a hole to the outer boundary of $D_{2}$ (i.e., to the unit circle). We denote this region ( $D_{2}$ minus cuts) by $D(1)$, and enumerate the cuts by $e(1, k, l), k=1, \ldots, n^{2}, l=1,2$ so that $e(1, k, 1)=e(1, k, 2)$ as sets, and as $z$ approaches $e(1, k, 1)$ the argument of $z$ increases. Let $D(j), j=1, \ldots, n$, be the copies of $D(1)$ with the corresponding cuts $e(j, k, l), j=1, \ldots, n, k=1, \ldots, n^{2}$, $l=1,2$. For all $j(\bmod n)$, paste $D(j)$ and $D(j+1)$ by identifying $e(j, k, 1)$ with $e(j+1, k, 2), k=1,2, \ldots, n^{2}$. The resulting surface is conformally equivalent to $R$ with the natural projection map $\pi \approx F_{2}$. By analytic continuation, the map 
$h^{-1} \circ L^{-1} \circ h_{2} \circ \pi$ is well-defined on $R$, hence analytic. We leave the details to the reader.

4. One can recover the same Riemann surface $R$ via interpolation problems. Fix $\epsilon \in\left(0, \frac{1}{2}\right)$ and let $D_{1}^{\prime}:=\{z: \epsilon<|z|<1\}$. Choose a natural number $n$ sufficiently large so that $2^{-n}<\epsilon$, and let $E_{n}$ be the set of $n$-th roots of $2^{-n}$. Note that $|z|=\frac{1}{2}$ for all $z \in E_{n}$.

We consider two interpolation problems:

(1) Find $G_{1} \in H^{\infty}\left(D_{1}^{\prime}\right)$ (with the smallest uniform norm) such that $G_{1}(z)=\bar{z}$ for all $z \in E_{n}$.

(2) Find $F_{2} \in H^{\infty}\left(D_{1}^{\prime}\right)$ (with the largest $\delta_{0}:=\min _{z \in D_{1}^{\prime}}\left\{\left|F_{2}(z)\right|,|z|\right\}$ ) such that $\left\|F_{2}\right\|=1$ and $F_{2}(z)=0$ for all $z \in E_{n}$.

Any solution $G_{1}$ of (1) has uniform norm greater than $C / \epsilon$, for some absolute constant $C$. To see this, one can repeat the argument in Section 2; thus, for $w$ such that $\epsilon^{n}<|w|<1$, define

$$
f(w)=\frac{1}{n} \sum z G_{1}(z)
$$

where the summation is over all $z \in D_{1}^{\prime}$ such that $(\epsilon / z)^{n}=w$. Note that $f\left(2^{n} \epsilon^{n}\right)=\frac{1}{4}$ since $z G_{1}(z)$ equals $\frac{1}{4}$ for $z \in E_{n}$, and then Cauchy's integral formula gives a lower bound estimate $\left\|G_{1}\right\| \geq C / \epsilon$.

On the other hand, any solution $F_{2}$ of (2) should yield a small $\delta_{0}=o(1)$ as $\epsilon \rightarrow 0$. To see this, let $F_{1}=z$ and $F_{2}$ be the solution of (2). Now if $\delta_{0}$ were not $o(1)$, the pair $\left(F_{1}, F_{2}\right)$ would become a set of corona data on $D_{1}^{\prime}$ with corresponding $0<\delta \leq$ $\liminf _{\epsilon \rightarrow 0} \delta_{0}$. But then any corona solutions $G_{1}$ and $G_{2}$ such that $F_{1} G_{1}+F_{2} G_{2}=1$ would have a bound $\geq C / \epsilon$, because $G_{1} / 4$ should be a solution of (1). This violates the corona theorem on annuli [Scheinberg 1963; Stout 1965]. (In fact, it violates a statement slightly stronger than the corona theorem, which is true for annuli; namely, for any annulus $D_{1}^{\prime}$ and corona data defined on $D_{1}^{\prime}$, there always exist corona solutions with bound $\leq M=M(\delta)$, where $M$ does not depend on $D_{1}^{\prime}$. See [Gamelin 1978, p. 47] for details.) Therefore to make $F_{1}$ and $F_{2}$ corona data, or to get a solution for (2) with large $\delta_{0}$, we take a number $N$ such that the multivalued function

$$
F(z)=\left(\frac{z^{n}-2^{-n}}{1-2^{-n} z^{n}}\right)^{1 / N},
$$

has modulus $\geq \frac{1}{4}$ for $|z|<\frac{1}{4}$. (Such an $N$ should be asymptotically greater than a fixed multiple of $n^{2}$ as $n \rightarrow \infty$, as we have seen in Section 2. Also note that $F^{N}$ is a solution for (2).) Now since $F$ is not analytic on $D_{1}^{\prime}$, we consider the Riemann surface of $F$ over $D_{1}^{\prime}$, which gives us the Riemann surface $R$ constructed in Section 2 (with $\delta=\frac{1}{4}$ ). 


\section{Acknowledgement}

The author deeply thanks Donald Marshall for his invaluable assistance with this work. He also thanks D. Drasin, A. Eremenko, T. Gamelin, M. Hayashi, and S. Rohde for their helpful suggestions and encouragement.

\section{References}

[Alling 1964] N. L. Alling, "A proof of the corona conjecture for finite open Riemann surfaces", Bull. Amer. Math. Soc. 70 (1964), 110-112. MR 28 \#209 Zbl 0124.04202

[Barrett and Diller 1998] D. E. Barrett and J. Diller, "A new construction of Riemann surfaces with corona”, J. Geom. Anal. 8:3 (1998), 341-347. MR 2000j:30076 Zbl 0956.30023

[Behrens 1970] M. Behrens, "The corona conjecture for a class of infinitely connected domains", Bull. Amer. Math. Soc. 76 (1970), 387-391. MR 41 \#825 Zbl 0197.11502

[Behrens 1971] M. F. Behrens, "The maximal ideal space of algebras of bounded analytic functions on infinitely connected domains", Trans. Amer. Math. Soc. 161 (1971), 359-379. MR 55 \#8380 Zbl 0234.46057

[Carleson 1962] L. Carleson, "Interpolations by bounded analytic functions and the corona problem”, Ann. of Math. (2) 76 (1962), 547-559. MR 25 \#5186 Zbl 0112.29702

[Gamelin 1970] T. W. Gamelin, "Localization of the corona problem", Pacific J. Math. 34 (1970), 73-81. MR 43 \#2482 Zbl 0199.18801

[Gamelin 1978] T. W. Gamelin, Uniform algebras and Jensen measures, London Mathematical Society Lecture Note Series 32, Cambridge University Press, Cambridge, 1978. MR 81a:46058 Zbl 0418.46042

[Garnett 1981] J. B. Garnett, Bounded analytic functions, Pure and Applied Mathematics 96, Academic Press, New York, 1981. MR 83g:30037 Zbl 0469.30024

[Garnett and Jones 1985] J. B. Garnett and P. W. Jones, "The corona theorem for Denjoy domains", Acta Math. 155:1-2 (1985), 27-40. MR 87e:30044 Zbl 0578.30043

[Hayashi 1999] M. Hayashi, "Bounded analytic functions on Riemann surfaces", pp. 45-59 in Aspects of complex analysis, differential geometry, mathematical physics and applications (St. Konstantin, 1998), edited by K. S. Stancho Dimiev, World Sci., River Edge, NJ, 1999. MR 2001i:30045 Zbl 0962.30023

[Jones and Marshall 1985] P. W. Jones and D. E. Marshall, "Critical points of Green's function, harmonic measure, and the corona problem", Ark. Mat. 23:2 (1985), 281-314. MR 87h:30101 Zbl 0589.30028

[Nakai 1982] M. Nakai, "Corona problem for Riemann surfaces of Parreau-Widom type", Pacific J. Math. 103:1 (1982), 103-109. MR 85c:30047 Zbl 0565.30028

[Scheinberg 1963] S. Scheinberg, Hardy spaces and boundary problems in one complex variable, Ph.D. thesis, Princeton University, 1963.

[Stout 1965] E. L. Stout, "Bounded holomorphic functions on finite Reimann surfaces", Trans. Amer. Math. Soc. 120 (1965), 255-285. MR 32 \#1358 Zbl 0154.32903

Received June 12, 2005. 


\section{BYUNG-GEUN OH}

Korea Institute for AdVANCED STUdy

207-43 CHEONGNYANGNI 2-DONG

DONGDAEMUN-GU

SEOUL 130-722

KOREA

bgoh@kias.re.kr 